\title{
Trial of VADs in children with HF
}

The utility of a ventricular assist device (VAD) as a bridge to transplantation has been prospectively assessed in children with heart failure (HF). VAD use was associated with substantially improved survival compared with the other circulatory support therapy available for pediatric use-extracorporeal membrane oxygenation (ECMO) - but was also associated with high rates of serious adverse events.

Although less prevalent in children than in adults, HF is associated with high mortality in the pediatric population, in part because donor hearts for children are in very short supply. At present, ECMO is typically used as a bridge to transplantation in small children with $\mathrm{HF}$, but is associated with serious adverse effects and so is usually only used for 10-20 days-a period that is much shorter than current median waiting times for donor organs. As a result, only about half of children who need support with ECMO survive long enough to receive a replacement heart. The Berlin Heart Study investigators, therefore, set out to prospectively test whether a VAD currently available in a wide range of sizes would be a useful alternative for bridge to transplantation in children with HF.

The Excor ${ }^{\circledast}$ Pediatric pulsatile-flow VAD (Berlin Heart GmbH, Berlin, Germany) was implanted into 48 children (aged $<16$ years; 3-60 kg) waiting for heart transplantation. VAD size was determined on the basis of age and body weight. For the analysis of outcomes, the patients were stratified into two cohorts according to body-surface area (BSA). Outcomes for cohort 1 ( $n=24$; BSA $<0.7 \mathrm{~m}^{2}$; median age 1 year) and cohort $2\left(n=24\right.$; BSA $0.7 \mathrm{~m}^{2}$ to $<1.5 \mathrm{~m}^{2}$; median age 9 years) were compared with propensity-score-matched historical control groups (each $n=48$ ) who had received ECMO.

In the VAD cohorts, the primary efficacy end point was time from device implantation to death, or to weaning from the VAD in patients who experienced unacceptable neurological outcomes (coma or profound sensory, motor, language, or cognitive impairment) after weaning. For patients who underwent heart transplantation after VAD use, or who experienced ventricular recovery and were weaned from the device without death or unacceptable neurological outcomes within 30 days of weaning, data were censored at the time of transplantation or weaning.

Neurological status was not available for individuals in the matched historical control cohorts, so the only primary efficacy end point for the controls was time to death. For patients who underwent device explantation and survived for $\geq 30$ days, either because of ventricular recovery or because they underwent heart transplantation, data were censored at the time of device explantation.

Median time on circulatory support was 28 days for VAD cohort 1, 43 days for VAD cohort 2, and 5 days for both of the ECMO control groups. Time to the primary efficacy end points was considerably longer in the VAD groups compared with their matched controls. Median time had not been reached by 174 days in VAD cohort 1 but was 13 days in the matched

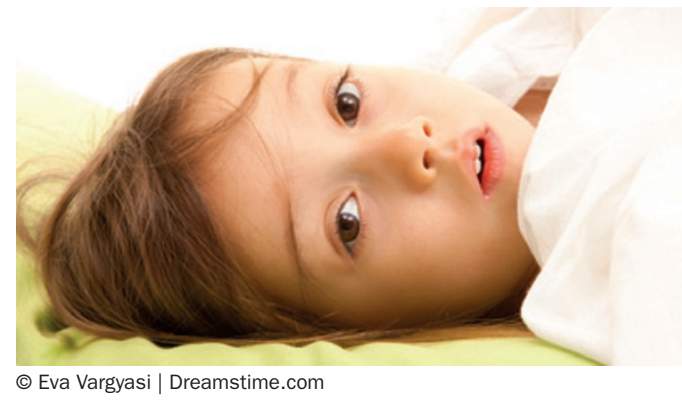

ECMO control group, and was 144 days for VAD cohort 2 versus 10 days for the matched controls.

In VAD cohorts 1 and 2, serious adverse events during circulatory support occurred in $92 \%$ and $79 \%$ of patients, respectively, and at rates of 0.07 (95\% CI 0.06-0.08) and 0.08 (95\% CI 0.06-0.09) events per patientday, respectively. The most common adverse events were infection (63\% and $50 \%$, respectively), major bleeding (42\% and 50\%), hypertension (50\% and $33 \%$ ), and stroke (29\% in both groups).

In an editorial that accompanied the report in the New England Journal of Medicine, Dr Linda Addonizio speculates that the strokes might reflect a problem with low-flow pulsatile systems and, therefore, that "perhaps [future] pediatric trials will show that small continuous-flow devices are effective, with lower complication rates". Nevertheless, on the basis of the available data, Dr Addonizio believes that "given the rates of neurologic complications...[VADs] should remain, at present, a last resort in small children."

Bryony M. Mearns

Original article Fraser, C. D. et al. Prospective trial of a pediatric ventricular assist device. N. Engl. J. Med. 367, 532-541 (2012) 